\title{
Pneumonia and influenza - Specific Considerations in Care Homes
}

\section{Adam Gordon $^{1}$ and Victoria Ewan ${ }^{2}$}

${ }^{1}$ Division of Rehabilitation \& Ageing, University of Nottingham Medical School, Queens Medical Centre, Nottingham, UK.

${ }^{2}$ Institute for Ageing and Health, Newcastle University, Newcastle upon Tyne, UK

\section{Correspondence to:}

Dr Adam Gordon

Clinical Lecturer in Medicine of Older People, Room B98,

Division of Rehabilitation \& Ageing,

University of Nottingham Medical School,

Queens Medical Centre,

Nottingham NG7 2UH.

Tel (direct): +44 (0) 1158230236

Mobile: +44 (0) 7939053776

Email: adam.gordon@nottingham.ac.uk

This paper has been accepted for publication and will appear in a revised form, subsequent to peer review and/or editorial input by Cambridge University Press, in Reviews in Clinical Gerontology published by Cambridge University Press. Cambridge University Press hold the copyright. 


\section{Summary}

This review provides an update on current evidence surrounding epidemiology, treatment and prevention of lower respiratory tract infection, with special reference to pneumonia and influenza, in care home residents. The care home sector is growing and provides a unique ecological niche for infections, housing frail older people with multiple comorbidities and frequent contact with healthcare services. There are therefore considerations in the epidemiology and management of these conditions which are specific to care homes. Opportunities for prevention, in the form of vaccination strategies and improving oral hygiene, may reduce the burden of these diseases in the future. Work is needed to research these infections specifically in the care home setting and this article highlights current gaps in our knowledge.

Keywords: Nursing home, influenza, pneumonia, cross-infection, aged 


\section{Introduction}

In most developed countries, 4 to $8 \%$ of people over the age of 65 years live in care homes (1), of whom 70 to $90 \%$ have some form of activity limitation (2-5). Around $40 \%$ of United Kingdom (UK) and United States (US) care home residents are completely bed bound $(4,6)$. Care homes vary in size, structure and purpose from country to country depending on the wider socio-political context in which they have developed, however they globally serve a frail population. In the UK, the term "care home" comprises residential and nursing homes.

Care home residents are susceptible to respiratory tract infections, with a prevalence of 0.5 to 4.4 per 1,000 resident days (7). Pneumonia, in particular, is important as the leading infective cause of death amongst care home residents, with mortality rates between $6 \%$ and $23 \%$ (8). In North America in 1993, assuming a prevalence of 1 per 1000 resident days, the direct hospital costs relating to an admission for pneumonia in a nursing home resident were estimated at $\$ 14,000$, which would exceed $\$ 8$ billion across 1.5 million nursing home residents (9).

There are specific considerations in the classification, epidemiology, diagnosis, management and prophylaxis of both pneumonia and influenza in the care home population that makes them worthy of special consideration. These will be considered in turn below. 
We searched Medline 1950 - December week 42009 using the terms ["nursing home acquired pneumonia" OR (Pneumonia AND Cross-infection AND Nursing homes) OR (Nursing home AND Influenza)]. Results were limited to English language articles; reviews other than systematic reviews were excluded. Sixty- four relevant articles were identified, of which 21 were duplicates and 43 were included in this review. Relevant papers already known to the authors but not identified using the above search strategy were also included.

\section{The terminology of infection in care homes is different}

Pneumonia refers to infections of the lung parenchyma that produce visible infiltrates on chest radiography, often associated with cough, fever, breathlessness and malaise. If there are no visible changes on the chest radiograph then the illness is called a lower respiratory tract infection (LRTI). It has been proposed that nursing home acquired pneumonia (NHAP) be considered separately from community acquired pneumonia (CAP) due to the likelihood that the causative organisms, and therefore the choice of antibiotic, will differ (10).

Most of the evidence surrounding NHAP comes from US studies and it is difficult to compare the prevalence of NHAP in US and non-US populations because of international variation in care homes and the medical therapies to which residents are exposed. For example, in the US, nursing home residents with severe pneumonia may be intubated and ventilated(11)(12) - which may both facilitate and 
cause higher diagnosis of drug-resistant pathogens. Such practice would be rare in the UK.

Healthcare associated pneumonia (HCAP) is a relatively new term which includes patients in nursing homes as being at higher risk of having pneumonia caused by multi-resistant organisms (13). Taking the UK as an example, a study conducted in 2001 compared the organisms causing community acquired pneumonia in nursing home residents with age and dependency matched controls living in their own home, demonstrating little difference between the two.(14) It is therefore unclear whether NCAP or HCAP are useful classifications in the UK.

The terminology surrounding influenza in care homes is the same as in the general population, but is described here to clarify discussion about influenza subtypes later in the article. Influenza refers to the infections caused by the influenza virus, of which there are three types, A, B and C, classified according to viral matrix- and nucleo-proteins. Of these, only A and B cause significant infection in humans. Influenza viruses are described according to their surface antigens, haemaglutinin and neuraminidase, which are glycoproteins known to play key roles in the replication cycle of the virus. Influenza A expresses 16 subtypes of haemaglutinin $(\mathrm{H} 1-\mathrm{H} 16)$ and 9 subtypes of neuraminidase (N1 -N9), whilst influenza B expresses only one type of each antigen. 


\section{The epidemiology of pneumonia and influenza in care homes is different from in the general population}

Care homes are unique ecological niches for infection because their residents have reduced function, have numerous comorbidities, and have higher rates of dementia. They live in communities in which they are grouped with other patients of similar age and vulnerability. A consequence of this is that the epidemiology of pneumonia and influenza in care homes, particularly in terms of outbreak management and infection control, is different from that in both the acute hospital and community sectors.

In general, mortality from pneumonia in care home residents is high (12-21\%) (1518), and residents are more likely to die of pneumonia than other healthcare associated infections (19). The higher mortality in residents is explained to a large extent by their pre-morbid status, with mortality rates attenuated compared to community controls when weight loss, dementia, aspiration risk and functional status are considered $(14,20)$.

Bacterial pneumonia is not generally regarded as contagious and isolation measures are therefore not routinely recommended. However, occasional outbreaks have been reported in care homes $(21,22)$, presumably because of the close proximity in which residents live. Where reported, outbreaks have been associated with low pneumococcal vaccination rates, making a compelling case for vaccination in this cohort, despite concerns about vaccine efficacy (see below). 
By contrast, influenza is characterised by epidemics. Influenza surface antigens frequently undergo structural modification, leading to a period when populations become vulnerable to infection whilst population immunity catches up. Subtle modification from season to season, usually caused by point mutation in the genetic code for the surface glycoproteins, is known as antigenic drift and is the driving factor behind seasonal influenza outbreaks. Rarely, entirely novel $\mathrm{H}$ or $\mathrm{N}$ antigens emerge, often as a consequence of genetic reassortment with swine or avian influenza viruses. This is known as antigenic shift and results in a strain to which there is limited or no population immunity, which often results in pandemic influenza. The concept of an "influenza season" comes from temperate latitudes in the northern and southern hemisphere, where characteristic peaks of influenza are recorded in the autumn and winter months. Care home outbreaks mirror the prevalence in the wider population and therefore tend also to be seasonal at these latitudes. In tropical climates, influenza is more common during the wet season but seasonal differences are less pronounced (23).

Once introduced into a care home, influenza can spread rapidly because of close contact among residents, their poor overall health status and the challenges of infection control in care homes. Rates of pneumonic conversion from influenza can be as high as $42 \%$ during outbreaks (24). During the influenza season, case-fatality rates for care home residents regularly exceed $5 \%$ and have been reported to exceed $70 \%$ during outbreaks (25-28). As with pneumonia, the risk of mortality or significant morbidity from influenza increases with age and co-morbidities (29) 
Spread of influenza is likely to occur via close personal contact, and in particular through contact with oral secretions (30). Staff represent a potential vector for spread through their role in mouth care and feeding. Recommended measures to minimise spread during an outbreak include regular handwashing for staff, ready availability of portable alcohol gel for hand cleansing and, where staff have prolonged close contact with residents, the use of face-masks, gloves and aprons (31-33). However the evidence for effectiveness of infection control in the care home setting is limited (34). This is possibly due to the fact that hospital-style measures are made difficult by the need to provide a homely setting and allow freedom of movement. Lack of dedicated infection control staff and low staff to resident ratios also contribute.

\section{Oropharyngeal commensals are different in the care home population}

Lower respiratory tract infections result from the combination of a potential pathogen adhering to the oro- or nasopharynx, the provision of an easy path to the lower respiratory tract (e.g. tendency to aspirate), and an immune system unable to eradicate the pathogen either at the oro- or nasopharynx or in the lungs. Age, number of comorbidities (35-37), malnutrition (38) and frailty influence the latter.

Micro-aspiration, where small amounts of oral-pharyngeal secretions are aspirated into the lungs, is likely to be an aetiological factor in most pneumonias and explains 
how differences in oral-pharyngeal colonisation lead to differences in the spectrum of organisms causing pneumonia in different populations $(39)(7,40)$.

Oral colonisation with pathogens known to cause pneumonia (e.g. P. aeruginosa, $S$. aureus ) occurs more commonly in hospitalised and institutionalised people (39) (7, 40). These bacteria are also encountered very commonly in those with nasogastric tubes in- situ $(41,42)$. In addition, both community and hospital acquired pneumonia have been associated with proton pump inhibitor prescription $(43,44)$ suggesting possible gastric bacterial overgrowth as an explanation for the presence of these bacteria.

In addition to guiding antibiotic prescribing for pneumonia in care home residents, these data suggest a possible role for manipulating the organisms that colonise the oropharyngeal tract as a way of modifying pneumonia risk in care home residents.

\section{Diagnosing pneumonia or influenza in care home residents is more difficult than in the general population}

Typical symptoms of lower respiratory tract infection (LRTI) include cough, fever, breathlessness, malaise, myalgia, and pleuritic pain. Viral infections may also cause upper respiratory tract symptoms such as sore throat and sneezing. As with many

older patients, the clinical presentation of pneumonia in care home residents is often non-specific because of general frailty, symptoms of comorbid illness, impaired 
communication and/or cognition, and altered response to sepsis (34). Fever, productive cough and pleuritic chest pain are less common in older people, whilst non-specific presentations such as falls, delirium, anorexia, or generalised weakness are common $(14,45)$.

The only English-language guideline for diagnosing pneumonia in care home residents comes from the US and is based upon consensus by a multi-specialty consensus panel (46). It suggests that a diagnosis of probable pneumonia can be made based on two of the following factors being present: new or worsening cough, new purulent sputum, fever $>100^{\circ} \mathrm{F}$, or over $2^{\circ} \mathrm{F}$ above normal, or $<96^{\circ} \mathrm{F}$, dyspnoea, respiratory rate $>25$, tachycardia, new or worsening hypoxia, pleuritic chest pain, decline in cognition or functional status, or new crepitations or wheezes heard on auscultation. Unlike other pneumonia guidelines, a chest radiograph is not required for diagnosis. The rationale for this is not made clear but, possibly, is a pragmatic decision based on the limited access to radiography in the care home setting and the possible deleterious effects of moving to an acute care setting to obtain an x-ray.

The presence of such symptoms is not, however, a reliable indicator of pneumonia, as opposed to other lower respiratory tract infections, nor does it give any indication as to the causative organism(24). Hui et al (47) found the aetiological agent in such presentations amongst care home residents to be bacterial in $53.3 \%$ of the cases where a pathogen was identified. Influenza virus type A or B caused $7.4 \%$ of cases. RSV, metapneumovirus and parainfluenza were all more prevalent than influenza. 
It is difficult to grade severity of pneumonia in older patients, including care home residents. The CURB-65 criteria (widely used in community acquired pneumonia) have a high negative predictive value (95.9\%) and sensitivity (85.2\%) but a low specificity (57\%) and positive predictive value (24.5\%) in community-dwelling older patients (48). This makes it easy to decide who has non-severe pneumonia but much more difficult to establish with certainty who has severe pneumonia and therefore requires hospitalisation - strict adherence to the CURB criteria will result in a significant number of unnecessary hospitalisations, particularly as high-quality care can often be provided within the care home setting.

Given the low sensitivity and specificity of clinical diagnosis (i.e. those with pneumonia at autopsy not displaying clinical symptoms or signs, and other respiratory illnesses being classified as pneumonia), a gold-standard investigation for pneumonia would be useful. Unfortunately, no such test is available. Inter-rater agreement between radiologists interpreting chest radiographs of nursing home residents is described as "fair" (49) and computed tomography does not reliably establish the diagnosis (50).

Influenza is diagnosed by clinical symptoms during epidemic or pandemic situations and by laboratory testing during outbreaks. Real-time polymerase chain reaction (PCR) analysis of nose and throat swabs in viral transport media allows same-day diagnosis to aid in infection control during outbreaks, although this is dependent on 
primary care physicians both making the provisional diagnosis and requesting the investigation.

\section{Mouth care may have a specific role in prophylaxis of LRTIs in care homes}

Given that the pathogenesis of NHAP appears to be microaspiration of infected oral secretions, it is possible that improving oral hygiene may reduce rates of pneumonia. Two systematic reviews have been undertaken considering oral hygiene and pneumonia. Scannapieco et al reviewed 21 studies and concluded that further research was needed but that evidence pointed to a link between the two (51). Azparnooh et al suggested a significant effect from oral hygiene, but included papers from a wider group of patients (e.g. intensive care and nursing home) and considered a wider range of diseases (52). Randomized controlled trials in this area are necessary.

One study considered in the reviews above is worth highlighting. Yoneyama et al (53) (17) randomised 418 nursing home residents to receive oral hygiene (manual toothbrushing plus povidone iodine oral scrubs) or no intervention, with the outcome measure being radiologically-confirmed pneumonia. 34 of 182 participants developed pneumonia in the no oral care group, compared to 21 of 184 in the oral care group OR $1.67(\mathrm{Cl} 1.01-2.75, \mathrm{p}=0.04)$. This study is the only intervention study in non-ventilated persons that is adequately powered and has not yet been repeated. 
Bassim et al subsequently undertook an intervention trial where one ward of patients was assigned an oral care practitioner and another ward was not (54). The intervention group had a similar rate of pneumonia at the end of the study but it was noted that this group would have been expected to have a higher rate given their higher prevalence of comorbidities. When adjusted for risk factors such as age, functional status, cognitive impairment and clinical suspicion of aspiration pneumonia, the odds ratio for death in the no oral care group was $3.57(p=0.3)$.

There is no evidence that feeding strategies modify pneumonia risk

Given that both aspiration and malnutrition represent risk factors for developing LRTIs and that both are common in the care home population, it seems logical to consider improving feeding strategies as a preventative intervention. Loeb et al conducted a systematic review of feeding interventions to prevent aspiration pneumonia in 2003 and retrieved 8 randomised controlled trials evaluating positioning changes, dietary interventions, pharmacologic therapies, oral hygiene, and tube feeding (55). None of the studies retrieved showed positive results, however most were statistically underpowered. Further work in this area has been suggested.

\section{Vaccination against pneumonia and influenza in care home residents is less effective than in the general population}


Streptococcus pneumoniae remains an important cause of NHAP and in the only organism with an available vaccine for older people. The World Health Organisation recommends that all adults over the age of 65 be vaccinated with the 23-valent Pneumococcal vaccine (PPV23). While the body of evidence available suggests a protective effect from the vaccine against invasive pneumococcal disease (56) (that is pneumococci detected from any site); the vaccine is less effective in older than younger people (57) and immunity wanes over time (58).

When endpoints such as hospitalisation for pneumonia are considered rather than invasive pneumococcal disease, the vaccine appears to be ineffective in older people (59). Huss et al recently re-analysed vaccine trials, and found that the vaccine conferred little protection once trials with inadequate blinding were excluded $(60,61)$. As stated earlier, the PPV23 vaccination may confer some herd immunity, reducing the likelihood of pneumococcal outbreaks in care homes, however the evidence for this is based around case reports. The risk-benefit ratio of pneumococcal vaccination in frail older people therefore remains uncertain and guidelines may be subject to revision in the future.

Similarly, most developed and many developing countries now recommend routine vaccination of older citizens against influenza (62). Whilst such guidelines necessarily include the care home population, the World Health Organisation guideline identifies care home residents as being at particularly high risk, and therefore representing a priority for influenza vaccination (63). 
Current recommendations are based on randomised controlled trials demonstrating efficacy of influenza vaccination which were conducted in young, healthy, ambulatory populations (64). No such work has been undertaken in the care home population and would now be considered unethical in the face of overwhelming expert consensus that vaccination should take place. Observational cohort studies, however, suggest that influenza vaccination in this group is effective and reduces the incidence of respiratory infection by $33-42 \%$ and the risk of pneumonia by $43 \%$ ( 65 , $66)$, this is compared with a reduction in the incidence of influenza of $78 \%$ in young fit subjects. Older patients are less responsive to vaccination(67), probably because of immunosenescence (68), and lower rates of seroconversion and seroprotection are seen following influenza vaccination in the elderly population compared with younger controls (69). However, despite its lower efficacy, observational studies suggest that homes with high uptake of influenza vaccination demonstrate a lower incidence of influenza-like illness $(26,65)$, again suggesting a possible role for herd immunity.

\section{Vaccination of staff against influenza is important in care homes}

Two large cluster-randomised controlled trials, and a Cochrane review drawn from them, have demonstrated that immunisation of staff has no effect upon incidence of influenza in care home residents but does reduce mortality, largely by reducing the incidence of complications $(25,70,71)$. This effect is only seen when residents are also immunised. No such data exists for staff vaccination against pneumococcus. 
Despite the demonstrated efficacy of staff vaccination, uptake rates for flu vaccines amongst health care workers are historically low. Only $16.5 \%$ of health care workers in England, for example, accepted vaccination in the 2008-2009 influenza season despite the vaccine being freely available and uptake being encouraged by employers (72). Immunisation rates in Dutch care home workers have been reported at $11 \%$ despite national guidelines recommending universal immunisation for staff (73).

Targeted interventions, including education programmes, financial incentives and reverse consent - where workers have to sign a consent form to demonstrate that they understand the risks taken by refusing vaccination - can improve vaccine uptake rates $(74,75)$. However, such practices are not widespread. Mandatory vaccination of care home workers, where employment is conditional upon vaccination status, has been suggested as a possible solution (76). The ethical challenges of such a policy are clear - balancing the need to protect patients from infection against the loss of autonomy for staff and the small possibility that they may suffer harmful side effects as a consequence of vaccination - and thus it remains the subject of debate rather than policy $(76,77)$.

The evidence-base and guidelines for chemoprophylaxis in care homes are different from the wider population 
Given the above comments about oral hygiene and feeding interventions, it is useful to consider pharmacological interventions to prevent aspiration pneumonia. El Solh et al conducted a systematic review of these in 2007 , considering angiotensin converting enzyme inhibitors, capsaicin, amantadine, cabergoline, theophylline and cilostazol (11). The latter four drugs could not be recommended due to adverse effects, and there was insufficient evidence to recommend the former two. The review concluded that further large scale randomised controlled trials were needed.

An earlier systematic review by Loeb et al (55) considered use of the drugs cilostazol and amantadine, and found that both lowered pneumonia incidence at 3 year followup, however state that both have a high incidence of undesirable side effects that limit their widespread use.

Neuraminidase inhibitors may be used to prevent influenza infection in exposed people. Indeed, the evidence for chemoprophylaxis is more convincing than that for treatment in care home residents. A randomized placebo-controlled trial of oseltamivir in 548 nursing home residents whose co-residents had laboratoryconfirmed influenza demonstrated reduced incidence of pneumonia, antibiotic prescribing and case mortality at 8 week follow-up(78). A similar randomized placebo-controlled trial of zanamivir in 494 residents demonstrated no effect on the incidence of laboratory confirmed influenza but a statistically significant $70 \%$ reduction in the incidence of influenza associated with pyrexial symptoms - possibly a clinically significant finding. (79) 
The UK National Institute for Clinical Effectiveness (NICE) recommends use of oseltamivir and zanamivir for post-exposure prophylaxis in at-risk people living in long-term residential or nursing homes, whether or not they are vaccinated (80). Similar guidance on prophylaxis has been issued by the US Centre for Disease Control and Prevention (CDC) (81).

Oseltamivir resistant strains of influenza have recently been detected in both the USA and Europe. Currently, this resistance is limited only to H1N1 strains (82). No zanamivir-resistant strains have been identified and it is postulated that the molecular structure of zanamavir means that it is fundamentally less likely to engender resistance. Oseltamivir is taken orally, whilst zanamivir is administered by inhalation using a diskhaler device. The inhalation route means that zanamavir is not recommended for use in patients with underlying lung disease, and some older patients have been shown to find loading and priming the inhaler difficult (83). Both of these factors limit its usefulness in the care home population.

\section{The choice of antibiotic therapy for pneumonia is more complex in the care home population}

Intravenous antibiotic therapy does not appear to confer a survival advantage in care home residents (20). Therefore residents can be treated in-situ with oral antibiotic therapy and only require admission to hospital where they are unable to take oral 
medications, require oxygen or intravenous fluids - which are not possible in the care home setting in many countries. The primary consideration in treating care home pneumonias is matching antibiotic therapy to the organisms prevalent in the care home population, as inadequate antibiotic therapy is associated with higher mortality(10).

The introduction of widespread antibiotic use has seen the emergence of less pathogenic bacteria causing respiratory infections in older people, including Staphylococcus aureus, coliform bacteria and Pseudomonas aeruginosa (84-86). Residents seem to be at particular risk of drug-resistant pneumonia if they have poorer functional status, have received antibiotics within the past 90 days or a recent hospital stay $(12,87)$.

This has led to revised antibiotic guidelines, in the US at least, for care home residents. New guidelines were issued by the American Thoracic Society in 2005 (88) covering HCAP which have been applied to nursing home residents hospitalised with NHAP and have changed recommendations to include antibiotic cover for the more unusual pathogens seen in this group.

El Solh et al compared outcomes of 334 nursing home residents hospitalised with NHAP/HCAP, of which 76 were treated using the new 2005 guideline and 258 were treated using the 2003 guideline, which did not include cover for MRSA or Pseudomonas aeruginosa (16). Despite the rationality of this approach, there was 
no difference in the time to clinical stability, in-hospital or 30-day mortality. This, combined with the uncertainty over whether pathogens in other countries mirror those seen in US nursing homes, means that standard CAP antibiotic protocols are used as first-line treatment for care home residents in many regions, with more broad-spectrum therapies used only if patients fail to respond to treatment.

\section{The guidelines for treating influenza in the care home population are different - although the evidence base for this is uncertain}

In treating patients with proven influenza, neuraminidase inhibitors have been shown to reduce the number of days until alleviation of symptoms, return to normal activity or resolution of fever. However, no specific treatment trials have been undertaken in the care home population and, where high-risk and elderly populations have been studied, the treatment effect, although present, has failed to achieve statistical significance(89). The elderly cohorts in these studies were small and, as a consequence, they were inadequately powered to measure complications as an outcome.

Despite the uncertainty about treatment effect, the UK National Institute of Clinical Excellence (NICE) regards the care home population as high-risk with respect to influenza and suggests that, when the virus is known to be circulating, treatment with oseltamivir should be commenced for residents with flu-like illness. Treatment is also supported for influenza-like illness in care home residents outside of influenza 
season but only when virological confirmation that influenza is the aetiological agent has been made (90).

These guidelines are not replicated elsewhere in the world and recent concern has focused on possible inconsistencies in the pharmaceutical research undertaken to evaluate oseltamivir, upon which NICE have based their recommendations (91). It is therefore possible, particularly given the paucity of care home specific data, that these guidelines will be subject to review in the future.

Oseltamivir has recently been the subject of much attention as a consequence of its heavy utilization for treatment of infected individuals in some regions during the current Swine-Origin Influenza A Virus (S-OIV) pandemic. The requirements for commencement in the context of a pandemic vary from country to country, as does the requirement for virological confirmation of infection by throat swab prior to commencement. In most cases, however, the elderly population, incorporating care home residents, are identified as a high risk group, where treatment can be commenced even in the absence of a pyrexia $(92,93)$.

\section{The threat posed by pandemic influenza is different in care homes than in the wider population}

A novel H1N1 Swine-origin influenza A virus (S-OIV) emerged in California and Mexico in April 2009(94) and has since spread around the world. The World Health 
Organisation (WHO) has defined this as a pandemic based upon its criterion of sustained human-to-human spread over multiple geographical regions(95).

At a population level, care home residents are potentially at lower risk from this virus than would conventionally be the case during a pandemic. Under 60 s make up the majority of serologically confirmed cases of influenza, with evidence that up to $33 \%$ of over 60s carry antibodies which are protective against S-OIV(96). This is likely to be a consequence of close similarities between the antigen profile of S-OIV and the strains of human H1N1 in prevalent circulation before the 1950s(97). At an individual level, however, care home residents remain at increased risk of secondary complications if they contract the virus because of their frailty and comorbidities.

Pandemic influenza will challenge care homes both as care providers and as organisations. Contingencies will be required to deal with increased care requirements amongst residents, for isolation of infected residents and to cover periods of staff absence(98). If homes are closed to new admissions during a pandemic, as is routine practice during normal influenza outbreaks, this will lead to loss of financial income, which will be compounded if the mortality rate is high. The importance of these issues varies from country to country, depending on the economic model underpinning the care home sector. In the UK, for example, care homes are predominantly run as small private companies which are particularly vulnerable to the economic pressures of a flu pandemic (98). In the USA, by contrast, nursing homes might be called on to deliver first-care if acute hospital facilities become overwhelmed, providing an additional stressor during a difficult time but also representing a possible revenue stream (99). There is evidence, however, 
that the care home sector in both countries has been engaged poorly in pandemic flu planning and, as a consequence, is potentially underprepared $(98,99)$.

Immunisation practices for care home residents during a pandemic will vary depending upon national policies. In the UK, for example, over 65s in high-risk groups, including the care home population, will be vaccinated as part of the first wave of vaccination(33). In the USA, by contrast, this group are not listed as high priority - with attention focusing instead on younger patients with comorbidities(100).

\section{Conclusions}

Both influenza and pneumonia cause considerable morbidity and mortality in care home residents. The care home population is different from the population at large in terms of age, co-morbidity, functional status and the communal environment in which they live. These differences result in differences in the behaviour of pneumonia and influenza, and how they are treated.

A number of issues remain unclear. It is not certain that differences between HCAP and CAP identified in US nursing home residents are apparent in care home settings in other countries. Nor is it clear that modifying antibiotic prescribing in US patients to allow for the differing spectrum of infection in HCAP makes any difference to clinical outcomes. Therefore the usefulness of this distinction remains uncertain. 
There is doubt that current diagnostic guidelines allow diagnosis of pneumonia in care home resident with any degree of specificity. Further work to identify reliable markers of pneumonic infection in this cohort would be welcome.

It is clear that aspiration of oropharyngeal commensals has some role, particularly, in the aetiology of nursing home acquired pneumonia and it is possible that this allows for improved oral care as a therapeutic intervention. Adequately powered randomized-controlled trial data in this area is very much needed.

Vaccination for influenza is indicated for both nursing home staff and residents, and is synergistic. There is no evidence-base to suggest that pneumococcal vaccination affects individual clinical outcomes in this cohort, although case reports suggest that it may convey protective herd immunity at a whole home level. Guidelines do not currently reflect this uncertainty.

Neuraminidase inhibitors are rapidly growing to become controversial therapies. Their use in prophylaxis in care homes is supported by independent randomizedcontrolled trials. However their role in treatment is less clear and guidelines may have to be reviewed to reconsider recent revelations about the quality of clinical evidence supporting the use of neuraminidase inhibitors in this respect. 
Pandemic influenza will challenge both the clinical and managerial skills of the care home sector. A pandemic will test the financial models of care homes at a time in which they are already facing considerable financial constraints. There is evidence that current planning may be insufficient and it is likely that further efforts are required to ensure preparedness.

Considerable effort is required, therefore, to establish an evidence-base for management of these infections in care homes. Whilst international guidelines and cross-national research may prove useful in this respect, the differences between care homes from country to country mean that one set of solutions may not suit all. The care home population is different from the population at large in a variety of ways with respect to pneumonia and influenza. We must understand these differences more thoroughly if we are to provide gold standard care to these patients.

\section{Conflicts of interest}

The authors declared no conflicts of interest 


\section{References}

1. Ribbe MW, Ljunggren G, Steel K, et al. Nursing Homes in 10 Nations: $A$ Comparison Between Countries and Settings. Age and Ageing. 1997;26(suppl_2):312.

2. Bajekal M. Health Survey for England 2000: Characteristics of Care Homes and Their Residents. London: The Stationary Office; 2002.

3. Falconer M, O'Neill D. Profiling disability within nursing homes: a censusbased approach. Age and Ageing. 2007;36(2):209-13.

4. US Centre for Disease Control and Prevention. National Nursing Home Survey. 2004 [cited 2009 30th November]; Available from:

http://www.cdc.gov/nchs/nnhs/nnhs products.htm.

5. Huang J-J, Lin K-C, Li I-C. Service needs of residents in community-based long-term care facilities in northern Taiwan. Journal of Clinical Nursing. 2008;17(1):99-108.

6. Bowman C, Whistler J, Ellerby M. A national census of care home residents. Age and Ageing, 2004:afh177.

7. Nicolle LE, McLeod J, Mclntyre M, MacDonell JA. Significance of pharyngeal colonization with aerobic gram-negative bacilli in elderly institutionalized men. Age and Ageing. 1986;15(1):47-52.

8. Nicolle LE, Strausbaugh LJ, Garibaldi RA. Infections and antibiotic resistance in nursing homes. Clinical Microbiological Reviews. 1996;9(1):1-17.

9. Terpenning M, Shay K. Oral health is cost-effective to maintain but costly to ignore.[comment]. Journal of the American Geriatrics Society. 2002;50(3):584-5. 
10. El-Solh AA, Sikka P, Ramadan F, Davies J. Etiology of severe pneumonia in the very elderly. American Journal of Respiratory \& Critical Care Medicine. 2001;163(3 Pt 1):645-51.

11. El Solh AA, Saliba R. Pharmacologic prevention of aspiration pneumonia: a systematic review. American Journal Geriatric Pharmacotherapy. 2007;5(4):352-62.

12. El Solh AA, Pietrantoni $\mathrm{C}$, Bhat $\mathrm{A}$, et al. Indicators of potentially drug-resistant bacteria in severe nursing home-acquired pneumonia. Clinical Infectious Diseases. $2004 ; 39(4): 474-80$.

13. Kollef MH, Shorr A, Tabak YP, et al. Epidemiology and outcomes of healthcare-associated pneumonia: results from a large US database of culture-positive pneumonia.Chest. 2005;128(6):3854-62.

14. Lim WS, Macfarlane JT. A prospective comparison of nursing home acquired pneumonia with community acquired pneumonia. European Respiratory Journal. $2001 ; 18(2): 362-8$

15. Degelau J, Guay D, Straub K, et al. Effectiveness of oral antibiotic treatment in nursing home-acquired pneumonia. Journal of the American Geriatrics Society $1995 ; 43(3): 245-51$.

16. El Solh AA, Akinnusi ME, Alfarah Z, et al. Effect of antibiotic guidelines on outcomes of hospitalized patients with nursing home-acquired pneumonia. Journal of the American Geriatrics Society. 2009;57(6):1030-5.

17. Yoneyama T, Yoshida M, Ohrui T, et al. Oral care reduces pneumonia in older patients in nursing homes. Journal of the American Geriatrics Society. $2002 ; 50(3): 430-3$.

18. Quagliarello V, Ginter S, Han L, et al. Modifiable risk factors for nursing homeacquired pneumonia. Clinical Infectious Diseases. 2005;40(1):1-6. 
19. Engelhart ST, Hanses-Derendorf L, Exner M, Kramer MH. Prospective surveillance for healthcare-associated infections in German nursing home residents. Journal of Hospital Infection. 2005;60(1):46-50.

20. van der Steen JT, Mehr DR, Kruse RL, et al. Treatment strategy and risk of functional decline and mortality after nursing-home acquired lower respiratory tract infection: two prospective studies in residents with dementia. International Journal of Geriatric Psychiatry. 2007;22(10):1013-9.

21. Nuorti JP, Butler JC, Crutcher JM, et al. An Outbreak of Multidrug-Resistant Pneumococcal Pneumonia and Bacteremia among Unvaccinated Nursing Home Residents. New England Journal of Medicine. 1998;338(26):1861-8.

22. Quick RE, Hoge CW, Hamilton DJ, et al. Underutilization of pneumococcal vaccine in nursing homes in Washington state: Report of a serotype-specific outbreak and a survey. The American Journal of Medicine. 1993;94(2):149-52.

23. Nicholson KG, Wood JM, Zambon M. Influenza. The Lancet. 2003;362(9397):1733-45.

24. Loeb M, McGeer A, McArthur M, et al. Surveillance for outbreaks of respiratory tract infections in nursing homes. Canadian Medical Association Journal. 2000;162(8):1133-7.

25. Hayward AC, Harling R, Wetten S, et al. Effectiveness of an influenza vaccine programme for care home staff to prevent death, morbidity, and health service use among residents: cluster randomised controlled trial. British Medical Journal. 2006;333(7581):1241-.

26. Serie C, Barme M, Hannoun C, et al. Effects of vaccination on an influenza epidemic in a geriatric hospital. Developments in Biological Standardization. 1977;39:317-21. 
27. Meiklejohn G, Hall H. Unusual outbreak of influenza A in a Wyoming nursing home. Journal of the American Geriatrics Society. 1987;35(8):742-6.

28. Saah AJ, Neufeld R, Rodstein M, et al. Influenza vaccine and pneumonia mortality in a nursing home population. Archives of Internal Medicine. 1986;146(12):2353-7.

29. Thompson WW, Shay DK, Weintraub E, et al. Influenza-Associated Hospitalizations in the United States. Journal of the American Medical Association 2004;292(11):1333-40.

30. Brankston G, Gitterman L, Hirji Z, Lemieux C, Gardam M. Transmission of influenza A in human beings. The Lancet Infectious Diseases. 2007;7(4):257-65. 31. Jefferson T, Foxlee R, Mar CD, et al. Physical interventions to interrupt or reduce the spread of respiratory viruses: systematic review. British Medical Journal. 2008;336(7635): 77-80.

32. Cowling BJ, Chan K-H, Fang VJ, et al. Facemasks and Hand Hygiene to Prevent Influenza Transmission in Households. Annals of Internal Medicine. $2009 ; 151(7): 437-46$.

33. Pandemic Influenza: Guidance for infection control in hospitals and primary care settings. London, Department of Health, 2007.

34. Nicolle L. Infection Control in Long Term Care Facilities. Clinical Infectious Diseases. 2000;31(3):752-6.

35. Harkness GA, Bentley DW, Roghmann KJ. Risk factors for nosocomial pneumonia in the elderly. American Journal of Medicine. 1990;89(4):457-63. 36. Lim WS, van der Eerden MM, Laing $\mathrm{R}$, et al. Defining community acquired pneumonia severity on presentation to hospital: an international derivation and validation study. Thorax. 2003;58(5):377-82. 
37. Fine MJ, Auble TE, Yealy DM, et al. A Prediction Rule to Identify Low-Risk Patients with Community-Acquired Pneumonia. New England Journal of Medicine. $1997 ; 336(4): 243-50$.

38. Morley JE, Silver AJ. Nutritional Issues in Nursing Home Care. Annals of Internal Medicine.1995;123(11):850-9.

39. Preston AJ, Gosney MA, Noon S, Martin MV. Oral flora of elderly patients following acute medical admission. Gerontology. 1999;45(1):49-52.

40. Russell SL, Boylan RJ, Kaslick RS, Scannapieco FA, Katz RV. Respiratory pathogen colonization of the dental plaque of institutionalized elders. Special Care in Dentistry. 1999;19(3):128-34.

41. Leibovitz A, Plotnikov G, Habot B, Rosenberg M, Segal R. Pathogenic colonization of oral flora in frail elderly patients fed by nasogastric tube or percutaneous enterogastric tube. Journals of Gerontology Series A-Biological Sciences \& Medical Sciences. 2003;58(1):52-5.

42. Leibovitz A, Plotnikov G, Habot B, et al. Saliva secretion and oral flora in prolonged nasogastric tube-fed elderly patients. Israel Medical Association Journal: 2003;5(5):329-32.

43. Herzig SJ, Howell MD, Ngo LH, et al. Acid-suppressive medication use and the risk for hospital-acquired pneumonia. Journal of American Medical Association. 2009;301(20):2120-8.

44. Laheij RJ, Sturkenboom MC, Hassing RJ, et al. Risk of community-acquired pneumonia and use of gastric acid-suppressive drugs. Journal of American Medical Association. 200427;292(16):1955-60.

45. Gavazzi G, Krause K-H. Ageing and infection. The Lancet Infectious Diseases. 2002;2(11):659-66. 
46. Hutt E, Kramer AM, Hutt E, Kramer AM. Evidence-based guidelines for management of nursing home-acquired pneumonia. Journal of Family Practice. 2002;51(8):709-16.

47. Hui DS, Woo J, Hui E, et al. Influenza-like illness in residential care homes: a study of the incidence, aetiological agents, natural history and health resource utilisation. Thorax. 2008;63(8):690-7.

48. Myint PK, Kamath AV, Vowler SL, Maisey DN, Harrison BDW. Severity assessment criteria recommended by the British Thoracic Society (BTS) for community-acquired pneumonia (CAP) and older patients. Should SOAR (systolic blood pressure, oxygenation, age and respiratory rate) criteria be used in older people? A compilation study of two prospective cohorts. Age and Ageing. 2006;35(3):286-91.

49. Loeb MB, Carusone SB, Marrie TJ, et al. Interobserver reliability of radiologists' interpretations of mobile chest radiographs for nursing home-acquired pneumonia. Journal of the American Medical Directors Association. 2006;7(7):416-9. 50. Masterton RG, Galloway A, French G, et al. Guidelines for the management of hospital-acquired pneumonia in the UK: Report of the working party on hospitalacquired pneumonia of the british society for antimicrobial chemotherapy. Journal of Antimicrobial Chemotherapy. 2008;62(1):5-34.

51. Scannapieco FA, Bush RB, Paju S. Associations between periodontal disease and risk for nosocomial bacterial pneumonia and chronic obstructive pulmonary disease. A systematic review. Annals of Periodontology. 2003;8(1):54-69.

52. Azarpazhooh A, Leake JL, Azarpazhooh A, Leake JL. Systematic review of the association between respiratory diseases and oral health. Journal of Periodontology. 2006;77(9):1465-82. 
53. Yoneyama T, Yoshida M, Matsui T, Sasaki H. Oral care and pneumonia. Oral Care Working Group. Lancet. 1999;354(9177):515.

54. Bassim CW, Gibson G, Ward T, et al. Modification of the risk of mortality from pneumonia with oral hygiene care. Journal of the American Geriatrics Society. 2008;56(9):1601-7.

55. Loeb MB, Becker M, Eady A, Walker-Dilks C. Interventions to prevent aspiration pneumonia in older adults: a systematic review. Journal of the American Geriatrics Society. 2003;51(7):1018-22.

56. Andrews RM, Counahan ML, Hogg GG, et al. Effectiveness of a publicly funded pneumococcal vaccination program against invasive pneumococcal disease among the elderly in Victoria, Australia. Vaccine. 2004;23(2):132-8.

57. Dominguez A, Salleras L, Fedson DS, et al. Effectiveness of pneumococcal vaccination for elderly people in Catalonia, Spain: a case-control study. Clinical Infectious Diseases. 2005;40(9):1250-7.

58. Konradsen HB, Konradsen HB. Quantity and avidity of pneumococcal antibodies before and up to five years after pneumococcal vaccination of elderly persons. Clinical Infectious Diseases. 1995;21(3):616-20.

59. Skull SA, Andrews RM, Byrnes GB, et al. Prevention of community-acquired pneumonia among a cohort of hospitalized elderly: benefit due to influenza and pneumococcal vaccination not demonstrated. Vaccine. 2007;25(23):4631-40. 60. Huss A, Scott P, Stuck AE, Trotter C, Egger M. Efficacy of pneumococcal vaccination in adults: A meta-analysis. Canadian Medical Association Journal. $2009 ; 180(1): 48-58$.

61. Jefferson T, Jefferson T. Pneumococcal vaccines: confronting the confounders. Lancet. 2009;373(9680):2008-9. 
62. van Essen GA, Palache AM, Forleo E, Fedson DS. Influenza vaccination in 2000: recommendations and vaccine use in 50 developed and rapidly developing countries. Vaccine. 2003;21(16):1780-5.

63. World Health Organization. Influenza vaccines: recommendations for the use of inactivated influenza vaccines and other preventive measures. Weekly Epidemiological Record. 2000;75:281-8.

64. Demicheli V, Di Pietrantonj C, Jefferson T, Rivetti A, Rivetti D. Vaccines for preventing influenza in healthy adults. Cochrane Database of Systematic Reviews 2007; (2): Available from:

http://www.mrw.interscience.wiley.com/cochrane/clsysrev/articles/CD001269/frame.h $\underline{\mathrm{tml}}$.

65. Monto AS, Hornbuckle K, Ohmit SE. Influenza Vaccine Effectiveness among Elderly Nursing Home Residents: A Cohort Study. American Journal of Epidemiology. 2001;154(2):155-60.

66. Ohmit SE, Arden NH, Monto AS. Effectiveness of inactivated influenza vaccine among nursing home residents during an influenza type A (H3N2) epidemic. Journal of the American Geriatrics Society. 1999;47(2):165-71.

67. Foster DA, Talsma A, Furumoto-Dawson A, et al. Influenza Vaccine Effectiveness in Preventing Hospitalization for Pneumonia in the Elderly. American Journal of Epidemiology. 1992;136(3):296-307.

68. Monto AS, Ansaldi F, Aspinall R, et al. Influenza control in the 21st century: Optimizing protection of older adults. Vaccine. 2009;27(37):5043-53.

69. Goodwin K, Viboud C, Simonsen L. Antibody response to influenza vaccination in the elderly: A quantitative review. Vaccine. 2006;24(8):1159-69. 
70. Carman WF, Elder AG, Wallace LA, et al. Effects of influenza vaccination of health-care workers on mortality of elderly people in long-term care: a randomised controlled trial. The Lancet. 2000;355(9198):93-7.

71. Thomas RE, Jefferson T, Demicheli V, Rivetti D. Influenza vaccination for healthcare workers who work with the elderly. Cochrane Database of Systematic Reviews. 2006; (3): Available from:

http://www.mrw.interscience.wiley.com/cochrane/clsysrev/articles/CD005187/frame.h $\underline{\mathrm{tml}}$.

72. Begum F, Pebody R. Summary report: influenza vaccination uptake for healthcare workers (HCWs) in England. Winter season 2008/09 at http://www.immunisation.nhs.uk/publications/FluVaccineUptake HCW Winter0809.p df.

73. Looijmans-van den Akker I, van Delden JJM, Hak E. Uptake of influenza vaccination in Dutch nursing home personnel following national recommendations. Journal of the American Geriatrics Society. 2007;55(9):1486-7.

74. Sand KL, Lynn J, Bardenheier B, Seow H, Nace DA. Increasing influenza immunization for long-term care facility staff using quality improvement. Journal of the American Geriatrics Society. 2007;55(11):1741-7.

75. Hofmann F, Ferracin C, Marsh G, Dumas R. Influenza Vaccination of Healthcare Workers: a Literature Review of Attitudes and Beliefs. Infection. 2006;34(3):142-7.

76. Jordan R, Hayward A. Should healthcare workers have the swine flu vaccine? British Medical Journal. 2009;339:b3398-.

77. van Delden JJM, Ashcroft R, Dawson A, et al. The ethics of mandatory vaccination against influenza for health care workers. Vaccine. 2008;26(44):5562-6. 
78. Peters PH, Gravenstein S, Norwood P, et al. Long-Term Use of Oseltamivir for the Prophylaxis of Influenza in a Vaccinated Frail Older Population. Journal of the American Geriatrics Society. 2001;49(8):1025-31.

79. Ambrozaitis A, Gravenstein S, van Essen GA, et al. Inhaled Zanamivir Versus Placebo for the Prevention of Influenza Outbreaks in an Unvaccinated Long-term Care Population. Journal of the American Medical Directors Association. 2005;6(6):367-74.

80. National Institute of Clinical Excellence. TA158 Influenza (prophylaxis) amantadine, oseltamivir and zanamivir: guidance. Available at http://guidanceniceorguk/TA158/Guidance/pdf/English (accessed 1st Nov, 2009). 81. USA Centre for Disease Control and Prevention. CDC Issues Interim Recommendations for the Use of Influenza Antiviral Medications in the Setting of Oseltamivir Resistance among Circulating Influenza A (H1N1) Viruses, 2008-09 Influenza Season, available at http://www2a.cdc.gov/HAN/ArchiveSys/ViewMsgV.asp?AlertNum=00279 (Accessed 30th October 2009).

82. Moscona A. Oseltamivir Resistance -- Disabling Our Influenza Defenses. New England Journal of Medicine. 2005;353(25):2633-6.

83. Diggory P, Fernandez C, Humphrey A, Jones V, Murphy M. Comparison of elderly people's technique in using two dry powder inhalers to deliver zanamivir: randomised controlled. British Medical Journal. 2001;322(7286):577-.

84. Garb JL, Brown RB, Garb JR, et al. Differences in etiology of pneumonias in nursing home and community patients. Journal of the American medical Association $1978 ; 240(20): 2169-72$. 
85. Kneeland Y, Jr., Price KM. Antibiotics and terminal pneumonia. A postmortem microbiological study. American Journal of Medicine. 1960;29:967-79.

86. Johanson WG, Dever LL. Nosocomial pneumonia. Intensive Care Medicine. 2003;29(1):23-9.

87. Brito V, Niederman MS, Brito V, Niederman MS. Healthcare-associated pneumonia is a heterogeneous disease, and all patients do not need the same broad-spectrum antibiotic therapy as complex nosocomial pneumonia. Current Opinion in Infectious Diseases. 2009;22(3):316-25.

88. American Thoracic Society, Infectious Diseases Society of America. Guidelines for the management of adults with hospital-acquired, ventilatorassociated, and healthcare-associated pneumonia. American Journal of Respiratory \& Critical Care Medicine. 2005;171(4):388-416.

89. Burch J, Corbett M, Stock C, et al. Prescription of anti-influenza drugs for healthy adults: a systematic review and meta-analysis. The Lancet Infectious Diseases. 2009;9(9):537-45.

90. National Institute of Clinical Excellence. TA168 Influenza - zanamivir, amantadine and oseltamivir (review): guidance. Available at http://http://guidanceniceorguk/TA168/Guidance/pdf/English (accessed 1st Nov 2009).

91. Cohen D. Complications: tracking down the data on oseltamivir. BMJ. 2009, 2009;339(dec08_3):b5387-.

92. UK Health Protection Agency. Pandemic flu: Clinical management of patients with an influenza-like illness during an influenza pandemic http://wwwhpaorguk/web/HPAwebFile/HPAweb C/1238055329071 (Accessed on Oct 30th, 2009). 
93. US Department of Health and Human Services. Updated Interim

Recommendations for the Use of Antiviral Medications in the Treatment and

Prevention of Influenza for the 2009-2010 Season.

http://wwwflugov/individualfamily/prevention/medicine/antiviralsrecommendhtml (accessed on 2nd Nov, 2009).

94. Novel Swine-Origin Influenza A Virus Investigation Team. Emergence of a Novel Swine-Origin Influenza A (H1N1) Virus in Humans. New England Journal of Medicine. 2009;360(25):2605-15.

95. World Health Organization. Pandemic Influenza Preparedness and Response: A WHO Guidance Document.

http://wwwwhoint/csr/disease/influenza/PIPGuidance09pdf (accessed 2nd Nov 2009).

96. Serum cross-reactive antibody response to a novel influenza $A(H 1 N 1)$ virus after vaccination with seasonal influenza vaccine. Morbidity and Mortality Weekly Report. 2009;58(19):521-4.

97. Peiris JSM, Poon LLM, Guan Y. Emergence of a novel swine-origin influenza A virus (S-OIV) H1N1 virus in humans. Journal of Clinical Virology. 2009;45(3):16973.

98. Fell G. Preparedness of Residential and Nursing Homes for Pandemic Flu. Journal of Public Health 2008;30(1):99-102.

99. Mody L, Cinti S. Pandemic Influenza Planning in Nursing Homes: Are We Prepared? Journal of the American Geriatrics Society. 2007;55(9):1431-7. 100. US Department of Health and Human Services. Vaccine Priority Groups. http://wwwflugov/individualfamily/vaccination/vprioritygroupshtml (accessed on 30th Oct, 2009). 
